[0212-7199 (2006) 23: 6; pp 279-281] ANALES DE MEDICINA INTERNA Copyright (C) 2006 ARAN EDICIONES, S.L.

AN. MED. InTERNA (Madrid) Vol. 23 , N. ${ }^{\circ}$ 6, pp. $279-281,2006$

\title{
Mixoma auricular izquierdo como forma de presentación de una angina de esfuerzo
}

\author{
P. J. MARCHENA YGLESIAS, L. P. DE BENITO CORDÓN, M. J. HERVÁS \\ LAGUNA, M. RUIZ CLIMENTE, J. F. DIMAS NÚÑEZ \\ Servicio de Medicina Interna. Hospital Virgen de la Luz. Cuenca
}

LEFT ATRIAL MYXOMA PRESENTING AS ANGOR PECTORIS

\section{RESUMEN}

Se presenta el caso de una paciente de 73 años sin factores de riesgo cardiovascular con angina y disnea de esfuerzo desde hacía 8 meses en el que el desencadenante fue un mixoma auricular izquierdo que protuía hacía la zona valvular y sin datos de obstrucción coronaria.

PALABRAS CLAVE: Mixoma auricular. Angor pectoris. Cardiopatía isquémica.

\begin{abstract}
A 73 years old woman without cardiovascular risk factors presented physical exercise angina and dyspnea since 8 months ago. The caused of the disease was a left atrial myxoma which entering in left ventricle. The patient was free of coronary injured.
\end{abstract}

KEY WORDS: Atrial myxoma. Angor pectoris. Ischemic cardiopathy.

Marchena Yglesias PJ, De Benito Cordón LP, Hervás Laguna MJ, Ruiz, Climente M, Dimas Núñez. JF. Mixoma auricular izquierdo como forma de presentación de una angina de esfuerzo. An Med Interna (Madrid) 2006; 23: 279-281.

\section{INTRODUCCIÓN}

Los tumores cardiacos primarios son una entidad poco frecuente. Mucho más frecuentes son las metástasis de tumores como la mama, el pulmón, los sarcomas de partes blandas, el melanoma, las leucemias y los linfomas (1) ,con una incidencia de alrededor del $10 \%$ (1) y de 20 veces los tumores primarios $(2,3)$. Éstos tienen una incidencia de entre 0,0017 y $0,3 \%$ en autopsias de pacientes no seleccionados (4). Un $75 \%$ de ellos son de estirpe benigna y de ellos el mixoma auricular representa alrededor del 50\% siendo el resto lipomas, fibroelastomas papilares y rabdomiomas. Se localizan en la aurícula izquierda en las tres cuartas partes de las ocasiones (1). La clínica clásica consiste en síntomas constitucionales, embolismos a distancia y obstrucción mitral simulando una estenosis. La manifestación como cardiopatía isquémica es excepcional $(5,6)$.

A continuación se describe el caso de una paciente con angina de esfuerzo y coronarias angiográficamente normales a la que se le diagnosticó un mixoma auricular izquierdo como causa de la cardiopatía isquémica.

\section{CASO APORTADO}

Mujer de 73 años de edad que como antecedentes personales refería únicamente un asma bronquial que trataba con broncodilatadores a demanda. No refería factores de riesgo cardiovascular. La paciente acudió al hospital por cuadro de hiperreactividad bronquial secundaria a infección respiratoria. En la anamnesis refería un dolor dorsal opresivo con sudoración y disnea desde hacía 8 meses que se desencadena con esfuerzos moderados, que cedía con el reposo y que en todo este tiempo no se había modificado. A la auscultación cardiaca estaba rítmica, presentaba un tercer ruido, un soplo sistólico y diastólico fluctuante y signos de insuficiencia cardiaca. En la auscultación pulmonar destacaban los roncus y sibilantes compatibles con la hiperreactividad bronquial que motivó el ingreso. La analítica mostraba anemia con aumento discreto de los reactantes de fase aguda. Los biomarcadores cardiacos fueron negativos. El ECG fue normal y en la radiografía de tórax se apreciaba una cardiomegalia, no presente en una previa de hacía un año. Con la sospecha inicial de hiperreactividad bronquial, angina de esfuerzo estable e insuficiencia cardiaca secundaria se solicitó un ecocardiograma que mostró una masa de 4 x $5 \mathrm{~cm}$ dependiente de la aurícula izquierda y que partiendo de la fosa oval protuía en diástole hacia el ventrículo produciendo una este-

Trabajo aceptado: 19 de diciembre de 2005 


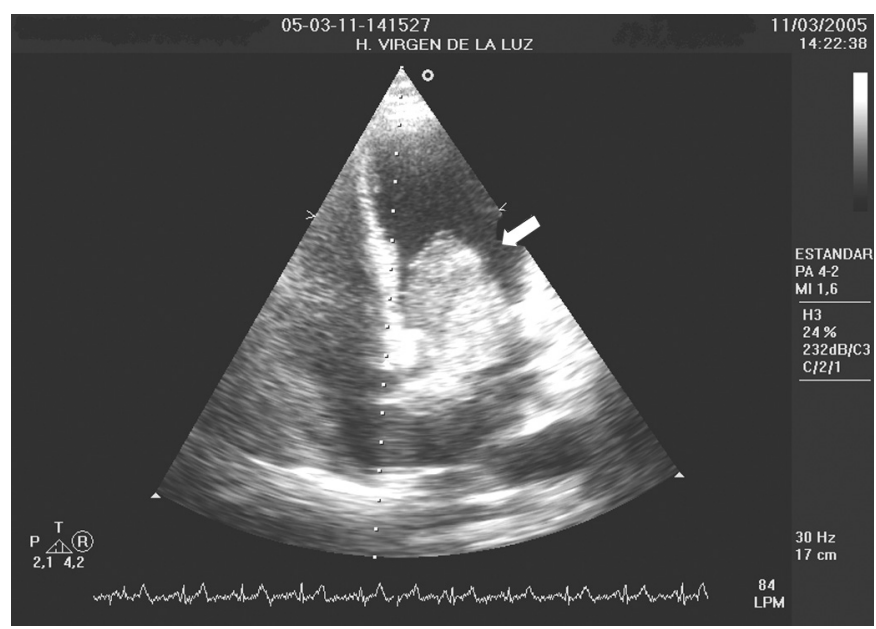

Fig. 1. Mixoma atrial izquierdo.

nosis mitral (Fig. 1). Posteriormente se realizó un cateterismo cardiaco en el que no se objetivaron lesiones coronarias angiográficamente significativas. Con la sospecha de mixoma auricular la paciente fue intervenida quirúrgicamente confirmándose el diagnóstico por la histología. Cuatro meses después de la intervención la paciente está asintomática y sin datos de recidiva.

\section{DISCUSIÓN}

Los mixomas auriculares constituyen los tumores cardiacos primitivos más frecuentes $(50 \%)$ dentro de la excepcionalidad de los mismos $(<0,3 \%$ en autopsias de pacientes no seleccionados) $(2,4)$.Se presentan entre los 30 y los 60 años con un predominio en las mujeres y la localización más frecuente es la aurícula izquierda (75\%) seguido de la aurícula derecha (15-20\%). Sólo un 4\% se localiza en los ventrículos (4). En un $90 \%$ de los casos son tumores solitarios pero pueden formar parte de síndromes familiares con afectación cutánea y endocrina. Se han propuesto múltiples acrónimos para estos síndromes (LAMB: léntigos, mixomas atriales, nevus azules, mixomas mucocutáneos; NAME: efélides, mixomas atriales, nevus, neurofibromatosis) pero recientemente se han englobado en una sola entidad conocida como síndrome de Carney que comprende además de lo anterior, los mixomas cutáneos, los mixomas o adenomas mamarios, la enfermedad adrenocortical nodular pigmentada, los tumores testiculares de células de Sertoli y adenomas hipofisarios secretores de hormona de crecimiento $(2,3,7)$.

Los mixomas son de estirpe histológicamente benigna. Derivan de las células mesenquimales multipotenciales del endocardio (3). En su mayoría son pedunculados, gelatinosos y friables lo que los hace extremadamente móviles condicionando parte de la clínica $(1,2,4)$. Son pocos los que cursan de forma asintomática, sobre todo si son de pequeño tamaño. La tríada clásica consiste en síntomas generales, embolizaciones a distancia y sintomatología obstructiva simulando una patología valvular. Los mixomas izquierdos cursan con insuficiencia cardiaca por ocupación tumoral y alto riesgo de enclavamiento en la mitral con síncope y muerte súbita. También cursan con embolización a las arterias coronarias, renales o cerebrales. Los derechos se manifiestan como insuficiencia cardiaca derecha, embolismo pulmonar e hipertensión pulmo$\operatorname{nar}(3)$.

Los síntomas generales (astenia, anorexia, fiebre, pérdida de peso, artromialgias, rash cutáneo, Raynaud, etc.) obligan a hacer un diagnóstico diferencial con conectivopatías, endocarditis infecciosa, fiebre reumática, vasculitis y procesos neoplásicos malignos (2). La embolización a distancia puede manifestarse como accidentes isquémicos cerebrales transitorios o episodios de cardiopatía isquémica.

Los embolismos coronarios son raros. Esto se explica por la localización del tracto de salida desde la aorta de las arterias coronarias que en la sístole están protegidas por los velos de la válvula aórtica (9). Su causa más frecuente son las endocarditis bacterianas (8). En la serie clásica de Wenger y Bauer se encontró una incidencia de embolismos por mixoma de $0,06 \%$ (8). Hay descritos 51 casos de infarto agudo de miocardio secundario a embolismo desde 1955 hasta 2002 (5,9). En 9 de 27 casos con coronariografía documentada ésta fue normal en el momento inicial y en 3 más en un estudio diferido en meses sin trombolisis previa por lo que varios autores sugirieron la posibilidad de una recanalización espontánea de las coronarias (9-12).

La obstrucción valvular es la tercera forma de manifestación clásica. Los mixomas izquierdos protruyen en diástole hacia la mitral ocasionando obstrucción del flujo auriculoventricular provocando síncope, muerte súbita así como compromiso del llenado ventricular y clínica de bajo gasto coronario (con angina) y episodios de insuficiencia cardiaca-edema agudo de pulmón como cualquier otra etiología de estenosis mitral (3).

El diagnóstico por medio del ecocardiograma transtorácico en 2D o transesofágico se han convertido en los métodos de elección si bien la tomografía o la resonancia magnética cardiaca puede constituir una prueba de imagen de indudable valor ya que proporcionan además cortes mediastinicos y de parénquima pulmonar. La angiografía ha decaído en los últimos años como técnica diagnóstica por el mayor riesgo embolígeno y por la alta sensiblidad y especificidad de las anteriormente mencionadas aunque es recomendable su realización para el estudio prequirúrgico y descartar lesiones coronarias concomitantes $(4,9)$.

Una vez diagnosticado, el tratamiento quirúrgico ha de ser precoz, siendo curativo en la mayoría de los pacientes, con un excelente pronóstico a largo plazo. Después de la resección se recomienda un control ecocardiográfico periódico ya que éstos puede recidivar hasta en un $2 \%$ de los casos esporádicos y un $22 \%$ en los casos familiares en los primeros 10 años tras la cirugía. La exéresis deficiente, el origen multifactorial del tumor, la implantación o movilización intraoperatoria y la embolización son las principales causas de las recurrencias $(4,5,13)$.

En resumen, los mixomas cardiacos constituyen una causa poco frecuente de cardiopatía isquémica. En nuestro caso la presencia de coronarias normales en la angiografía hace pensar en la hipótesis de un compromiso de llenado ventricular como causa de la angina y de la insuficiencia cardiaca ya que tras la exéresis del tumor la paciente ha permanecido asintomática realizando el mismo tipo de esfuerzo, pero sin poder descartar el origen embólico y la posterior lisis de los mismos recanalizándose precozmente las coronarias. 


\section{Bibliografía}

1. Butany J, Nair V, Naseemuddin A, Fair G, Catton C, Yau T. Cardiac tumours: diagnosis and management. Lancet Oncol 2005; 6: 219-28

2. Shapiro L. Cardiac tumours: diagnosis and management. Heart 2001; 85: 218-222.

3. Abad C. Tumores cardiacos. Generalidades. Tumores primitivos benignos. Rev Esp Cardiol 1998; 51: 10-20.

4. Reynen K. Cardiac myxomas. N Engl J Med 1995; 333: 1610-1617.

5. Abad C, Serra J, Condom E, Bosch X, Mestres CA, Paré JC. Infarto de miocardio en una mujer joven con un mixoma auricular izquierdo. Rev Esp Cardiol 1989; 42: 485-488.

6. Abascal V, Kasznica J, Aldea G, Davidoff R. Left atrial myxoma and acute myocardial infarction. A dangerous duo in the thrombolytic agent era. Chest 1996; 109: 1106-08

7. Morillas P, Frutos A, Bertomeu V, Valero R, Rodríguez JA, Climent V, Moragón M. Un caso de síndrome de Carney. Rev Esp Cardiol 2001; 54: $803-806$
8. Lehrman KL, Prozan G, Ullyot D, Atrial myxoma presenting as acute myocardial infaction. Am Heart J 1985; 110: 1293-5.

9. Braun S, Schrötter H, Reynen K, Schwencke C, Strasser RH. Myocardial infarction as complication of left atrial myxoma. Int $\mathrm{J}$ Cardiol 2005; 101: 115-121.

10. Hashimoto H, Takahashi H, Fujiwara Y, Joh T, Tomino T. Acute myocardial infarction due to coronary embolization from atrial myxoma. Jpn Circ J 1993; 57: 1016-20.

11. Rath S, Har-Zahav Y, Battler A, Agranat O, Neufeld NH. Coronary arterial embolus from atrial myxoma. Am J Cardiol 1984; 54: 1392-

12. Soejima Y, Niwa A, Tanaka M, Doi M, Nitta N, Takamoto T, et al. A left atrial myxoma complicated with acute myocadial infarction. Intern Med 1997; 36: 31-4.

13. Morillas PJ, Almenar L, Rueda J, Miró V. Tumores cardiacos primarios Estudio de 21 casos. Med Clin (Barc) 1998; 110: 356-58. 